\title{
Disziplin, Profession und evidenzbasierte Praxis: Zur Stellung der Psychotherapie im Gesundheitssystem. Eine Bilanz
}

\author{
Wilfried Datler · Markus Hochgerner · Christian Korunka · Henriette Löffler-Stastka · Gerhard Pawlowsky
}

Angenommen: 27. Januar 2021 / Online publiziert: 22. Februar 2021

(c) Der/die Autor(en) 2021

Zusammenfassung 30 Jahre nach der Verabschiedung des österreichischen Psychotherapiegesetzes wird die Besonderheit der Psychotherapie im Gesundheitssystem mit Bezug auf nationale Gegebenheiten und international vorliegende Forschungsergebnisse dargestellt. Dabei werden zentrale Qualitäts- und Unterscheidungsmerkmale der Psychotherapie im Verhältnis zur Klinischen Psychologie und zu weiteren Gesundheitsberufen unter Miteinbeziehung von evidenzbasierter Forschungsliteratur vorgestellt. Es wird deutlich gemacht, dass Psychotherapie das bedeutsamste und weitreichendste Versorgungsangebot zur interaktionellen Behandlung psychischer Erkrankungen darstellt. $\mathrm{Zu}$ den zentralen Merkmalen der Psychotherapie zählen die umfassenden, evidenzbasierten sowie wissenschaftstheoretisch und methodisch in vier Clustern verankerten Konzepte zur Erklärung, Diagnose und individuellen, prozessorientierten Behandlung von psychischen Erkrankungen sowie die im Vergleich zu ähnlichen Berufen deutlich höhere Qualität der Ausbildung.

Schlüsselwörter Psychotherapie $\cdot$ Klinische Psychologie - Psychiatrie und Psychotherapeutische Medizin · Ausbildung · Kompetenz

Die Nennung der AutorInnen erfolgt in alphabetischer Reihenfolge. Als korrespondierende Autorin fungiert Henriette Löffler-Stastka.

\section{H. Löffler-Stastka (ه)}

Klinik für Psychoanalyse und Psychotherapie, und Postgraduate Unit | Teaching Center, Medizinische Universität Wien, Währinger Gürtel 18-20, 1090 Wien, Österreich

henriette.loeffler-stastka@meduniwien.ac.at
Discipline, profession and evidence-based practice: The position of psychotherapy in the health care system. A balance

Summary 30 years after the implementation of the Austrian Psychotherapy Act, the distinctions of psychotherapy in the health system is presented with reference to national circumstances and international research results. In the following, essential quality and distinguishing features of psychotherapy in relation to psychology/clinical psychology and other health professions are presented on the basis of evidence-based research literature. Psychotherapy is the most important and provides the most extensive range of services for the interactional treatment of mental illnesses. The central features of psychotherapy include the comprehensive, evidence-based, and scientific theoretically and methodologically anchored concepts for explanation, diagnosis and individual, process-oriented treatment of mental illnesses, as well as the significantly higher quality of training compared to similar occupations.

Keywords Psychotherapy · Clinical Psychology • Psychiatry and Psychotherapeutic Medicine · Training · Competence

\section{Vorbemerkung}

Vor 30 Jahren wurde im österreichischen Parlament nach jahrzehntelangem Ringen das Psychotherapiegesetz verabschiedet (Kierein et al. 1991; Wißgott 2009). Obgleich wesentliche Beiträge zur Entwicklung der modernen Psychotherapie bereits im ausgehenden 19. Jahrhundert in Wien erarbeitet worden waren, bedurfte es damit etwa eines Jahrhunderts, ehe Psychotherapie als „eigenständiges Heilverfahren im Gesundheitsbereich für die Diagnostik und 
Behandlung von psychischen, psychosozialen oder auch psychosomatisch bedingten Leidenszuständen und krankheitswertigen Störungen“ gesetzliche Anerkennung fand (Bundesministerium 2019, S. 44). Psychotherapeut oder Psychotherapeutin durften sich ab dem Inkrafttreten des Gesetzes im Jahre 1991 nur mehr Personen nennen, die in die „Psychotherapeutenliste“ eingetragen waren. Dafür ist seit dem Auslaufen von Übergangsbestimmungen der Abschluss des psychotherapeutischen Propädeutikums und des psychotherapeutische Fachspezifikums im Sinne des Psychotherapiegesetzes nötig.

Die Umsetzung des Gesetzes, das 1991 in Kraft trat, zog zahlreiche Entwicklungen nach sich. Diese betrafen etwa die Gestaltung der Aus- und Weiterbildung, die Führung der Liste der eingetragenen PsychotherapeutInnen, die Einrichtung des Psychotherapiebeirats, den Ausbau der psychotherapeutischen Versorgung Österreichs oder die wissenschaftliche Befassung mit Psychotherapie. Diskussionen um die öffentliche Finanzierung der Psychotherapie intensivierten überdies Forderungen nach weiteren wissenschaftlichen Nachweisen, welche die Wirksamkeit des Einsatzes psychotherapeutischer Methoden betreffen.

Parallel dazu kam es auch in zwei Nachbardisziplinen zu einer verstärkten Befassung mit Psychotherapie und verwandten Tätigkeitsbereichen: Im Bereich der Psychologie wurden im Sinne des Psychologengesetzes postgraduale Ausbildungsgänge eingerichtet, in denen AbsolventInnen des Diplom- oder Masterstudiums der Psychologie zu Klinischen PsychologInnen qualifiziert werden (vgl. Psychologengesetz 2013). Und im Bereich der Medizin fanden drei Annäherungen an Psychotherapie statt: Psychotherapie erhielt im grundständigen Studium der Medizin einen größeren Stellenwert (Firbas 2001). Über die Österreichische Ärztekammer (ÖÄK) werden drei Weiterbildungsgänge für ÄrztInnen angeboten, die mit der Verleihung der PSY-Diplome für psychosoziale Medizin (PSY1), psychosomatische Medizin (PSY2) und psychotherapeutische Medizin (PSY3) abschließen (Ö̈̈K, ÖGPPM o.J.). Und der Facharzttitel „Psychiatrie“ wurde auf „Psychiatrie und Psychotherapeutische Medizin“ erweitert und zog eine veränderte Ausbildungsordnung (ÖÄK 2018) nach sich.

Angesichts dieser Entwicklungen ist es angebracht, sich der Psychotherapie im Sinne des österreichischen Psychotherapiegesetzes zuzuwenden und im Wissen darum Bilanz zu ziehen, dass eine Neufassung des Psychotherapiegesetzes zur Diskussion steht (vgl. Datler et al. 2021). Unter Einbeziehung internationaler Forschungsbefunde werden punktuelle Vergleiche mit ausgewählten Aspekten der Klinischen Psychologie und der Psychotherapeutischen Medizin gezogen, zu welcher die FachärztInnenausbildung (Brittlebank et al. 2016; Union Europeenne des Medecins Specialistes UEMS 2017/18) qualifiziert.
Die angeführten Aspekte stehen notwendigerweise in einem engen Zusammenhang mit nationalen Gegebenheiten, sind zugleich aber auch in internationale Diskurse (vgl. European Association for Psychotherapy 2013) eingebettet, die in den letzten Jahrzehnten ebenfalls an Dynamik zugenommen haben. Es ist daher 30 Jahre nach der Beschlussfassung über das österreichische Psychotherapiegesetz angebracht, auf einige der oben genannten Aspekte näher einzugehen.

\section{Zur evidenzbasierten Wirksamkeit psychotherapeutischer Behandlungen}

Psychotherapie zeichnet sich durch ausgesprochen hohe Behandlungswirksamkeit aus (mittlere Effektstärken in den großen Metaanalysen zwischen 0,5 und 0,7; z. B. Cooper et al. 2013; Cuijpers et al. 2020). Im Vergleich ist Psychotherapie damit einer pharmakologischen Behandlung zumindest ebenbürtig, einigen Metaanalysen zufolge, zum Teil sogar deutlich, überlegen (z. B. Cooper et al. 2013). Eine Kombination aus beiden Behandlungskonzepten ist oft besonders wirksam (Cuijpers et al. 2020). Die hohe Wirksamkeit lässt sich für unterschiedliche Störungsbilder und für unterschiedliche Therapieverfahren zeigen (z. B. Lambert 2013).

Dabei zeichnet sich Psychotherapie besonders durch eine langfristige und nachhaltige Behandlungswirkung aus (Zimmermann et al. 2015), woraus eine beachtenswerte Kosteneffizienz resultiert (De Maat et al. 2013). Dies gilt auch für hochfrequente, über einen längeren Zeitraum durchgeführte Behandlungen. So konnte etwa in einer von der Medizinischen Universität Wien 2014 vorgelegten MetaAnalyse, in welche die Auswertung von insgesamt 7000 Behandlungen aus 13 Katamnesestudien, 9 quasi-experimentellen Studien und 4 randomisiert-kontrollierten Studien eingingen, gezeigt werden, dass hochfrequente Psychoanalyse ein wirksames Psychotherapieverfahren darstellt, das bei einem Großteil der PatientInnen zu signifikanten und anhaltenden Verbesserungen des Gesundheits- und Krankheitszustandes führt. Belege aus randomisiert-kontrollierten Therapiestudien zeigen in diesem Zusammenhang, dass die Effekte der psychotherapeutischen Behandlung (anders als in den Kontrollgruppen) auch nach Ende der Behandlung weiter zunehmen (vgl. Zimmermann et al. 2015; De Maat et al. 2009).

Aus differenzierten Meta-Analysen der verfügbaren Kosten-Effektivitäts-Studien (De Maat et al. 2007, 2013) geht hervor, dass Psychotherapie zu einer nachhaltigen Senkung der Gesundheitskosten führt. Diese Reduktion entsteht durch die verminderte Inanspruchnahme medizinischer Leistungen und einer geringeren Anzahl von Arbeitsunfähigkeitstagen (vgl. Seitz et al. 2019). Die Kosten für die psychotherapeutischen Behandlungen amortisieren sich nach ca. 3 Jahren. 
Die neuere Wirkfaktorenforschung hat gezeigt, dass allgemeine Wirkfaktoren, wie die Person der TherapeutIn und die therapeutische Beziehung (vgl. Norcross und Lambert 2019; Norcross und Wampold 2019), eine besonders große und hilfreiche Bedeutung für den Therapieerfolg haben. Diese allgemeinen Wirkfaktoren haben einen stärkeren Einfluss auf den Therapieerfolg als spezifische Interventionstechniken. Dies korrespondiert mit dem Umstand, dass Psychotherapie seit jeher einen besonderen Schwerpunkt auf die Entwicklung dieser handlungsleitenden Wirkfaktoren sowohl in der psychotherapeutischen Theorie als auch in der Ausbildung und in der therapeutischen Praxis legt. Sie ist daher in ihrer Wirksamkeit Zugängen weit überlegen, die ihren Schwerpunkt vorrangig und handlungsleitend auf spezifische Interventionstechniken legen, die im Anschluss an eine vornehmlich ICD-basierte Diagnostik zum Einsatz kommen (wie dies beispielsweise in der klinischen Psychologie der Fall ist).

In der empirischen Psychotherapieforschung werden seit Jahrzehnten umfangreiche Studien durchgeführt, die eine nachhaltige Wirksamkeit der psychotherapeutischen Methoden bestätigt, die den vier psychotherapeutischen Grundströmungen respektive Clustern zugeordnet werden können. Diesen Clustern sind die psychoanalytisch/psychodynamischen (PPT), verhaltenstheoretisch orientierten (VT), humanistischen (HPT) und systemischen (SPT) psychotherapeutischen Verfahren zuzurechnen, von denen in Summe 23 in Österreich Anerkennung gefunden haben.

Die relevanten empirischen Studien umfassen realitätsnahe Feldstudien mit großen Fallzahlen (z. B. Stiles et al. 2008 für kognitive, humanistische und psychodynamische Behandlungsansätze), aber auch zahlreiche Studien nach dem sogenannten „Gold Standard“ (experimentelle Kontrollgruppendesigns, Meta-Analysen z. B. Cuijpers et al. 2008a, 2008b, 2020). Die vier psychotherapeutischen Grundströmungen (Cluster) weisen dabei ähnlich hohe Effektstärken auf (Lambert 2013).

Zusätzlich zu der im „Handbook of Psychotherapy and Behavior Change“ (Lambert 2013) sehr umfassend dargestellten und nach den Wirkfaktoren gegliederten Listung der empirischen Psychotherapieforschungsstudien aller therapeutischen Traditionen (PatientInnenfaktoren, TherapeutInnenvariable, Therapeutische Beziehung, allgemeine/unspezifische Wirkfaktoren, Technik) werden im Folgenden Forschungsergebnisse referiert, die fünf bedeutsame und häufig thematisierte Dimensionen betreffen, wobei vor allem Ergebnisse aus empirischen Untersuchungen mit berücksichtigt werden, die in Österreich durchgeführt oder unter Beteiligung österreichischer Forschungsgruppen realisiert wurden.

- Outcome: In zahlreichen Outcome-Studien wurde die Wirksamkeit von Psychotherapie in Hinblick auf alle vier Cluster nachgewiesen: Unter Bezugnahme auf die meisten psychiatrischen Erkrankungen referieren Leichsenring et al. (2015) und Shedler (2010) Belege für die Wirksamkeit psychoanalytisch-psychodynamischer Psychotherapien (Cluster 1/PPT). Die theoretische Basis, auf der PPT seit den Anfängen bei Freud basiert, ist von umfassender Literatur gestützt (Westen 1998; Westen et al. 2004). Für die verhaltenstherapeutischen Methoden (Cluster 2/VT) fassen Cuijpers et al. (2008a, 2008b) Outcome Studien mit Bezug auf die häufigsten psychiatrischen Störungsbilder in Metaanalysen zusammen. Nach Cain (2016) sowie Wampold und Imel (2015) wurden ähnliche Ergebnisse wiederholt und umfassend für den Bereich der Humanistischen Therapieverfahren (Cluster 3/HPT) nachgewiesen. Lambert (2013) referiert ähnliche Ergebnisse für systemische Therapiemethoden (Cluster 4/SPT).

- Nachhaltigkeit: Leichsenring und Rabung (2011) haben bezüglich Cluster 1 (PPT) gezeigt, dass Psychotherapie während und nach der Therapie langfristig wirkt, insbesondere in Hinblick auf Persönlichkeitsveränderungen über das Therapieende hinaus (siehe dazu auch Fonagy und Kächele 2009; Fonagy et al. 2015; Leuzinger-Bohleber et al. 2003, 2019; Zimmermann et al. 2015).

- Effektstärken: Manchen Untersuchungen zufolge erweisen sich psychotherapeutische Verfahren der Cluster 1 (PPT), 2 (VT) und 3 (HPT) als ähnlich wirksam wie andere Therapien (Steinert et al. 2017). Betrachtet man alleine die Effektstärken der therapeutischen Technik, ist der PPT-Prototyp wirksamer als der VT-Prototyp (Ablon und Jones 1998, 2005; Jones 2000; Zimmermann et al. 2015), der HPT-Prototyp gleich effektiv (Steinert et al. 2017).

- Naturalistische Studien: Stiles et al. (2008) vergleicht psychotherapeutische Verfahren der Cluster 1 (PPT), 2 (VT) und 3 (HPT) im Versorgungskontext und beschreibt Gleichwertigkeit bezüglich der Versorgungssituation. In der praxisorientierten Forschung und den naturalistischen Studien (De Maat et al. 2007; Leichsenring 2004; Riess 2018) wird durchgängig ein hoher Anteil der TherapeutInnenvariable an der Outcomevarianz beschrieben ( $17 \%$ vs. $5 \%$ in randomisierten kontrollierten Untersuchungen [RCTs]). Auch tragen naturalistische empirische Studien (z.B. auf Cluster 1 [PPT] oder 3 [HPT] bezogen) zu einem besseren Verständnis für psychische Prozesse und ihre Entwicklung bei (Blatt und Shichman 1983; Blatt 1995; Fonagy und Target 1997; Löffler-Stastka et al. 2008, 2010, 2018; Luyten und Blatt 2013; zu naturalistischen versus RCT-Studien siehe Fischer 2011, S. 121 ff.).

- Kosteneffizienz: De Maat et al. $(2007,2013)$ fassen Kosteneffizienzstudien zusammen und argumentieren v.a. mit der erwiesenen Nachhaltigkeit der psychotherapeutischen Behandlungen. Vergleiche mit pharmakologischen Behandlungen (Cuijpers et al. 2020) weisen der Psychotherapie eine deutlich 
höhere Kosteneffizienz aus. Untersuchungen der Sekundärkosten (Seitz et al. 2019) belegen ebenfalls die Vorteile von Psychotherapie.

Seit mehr als drei Jahrzehnten werden empirische Studien diskutiert, denen zufolge unterschiedliche psychotherapeutische Ansätze eine ähnliche Wirksamkeit haben (Luborsky et al. 1975; Luborsky 1995). Eine mögliche Erklärung dieser Untersuchungsergebnisse nimmt darauf Bezug, dass die meisten Effekte psychotherapeutischer Behandlungen durch allgemeine (unspezifische) Faktoren und nicht durch bestimmte Techniken (Cuijpers 1998) erzielt werden. $\mathrm{Zu}$ diesen Faktoren gehören verschiedene Aspekte der therapeutischen Beziehung zwischen TherapeutIn und PatientIn (Lambert 2004; Norcross und Lambert 2019; Norcross und Wampold 2019).

Über die Bedeutung der therapeutischen Beziehung für den Behandlungsverlauf besteht mittlerweile ein therapieschulen-übergreifender Konsens (Krause 1997; Margraf und Brengelmann 1992; Orlinsky und Howard 1986; Orlinsky et al. 1994; Rudolf 1991), wobei zwischen Konzepten zur Erklärung der Wirksamkeit von Psychotherapie unterschieden wird, in denen vor allem unspezifische oder aber spezifische Faktoren als entscheidend für den Therapieerfolg ins Treffen geführt werden (Butler und Strupp 1986). In den Konzepten, in denen der Fokus auf unspezifische Ansätze gelegt werden, ergibt sich die Bedeutsamkeit der Beziehung aus dem durch die Behandlungskonzeption definierten Rahmen und beinhaltet Variablen wie die Motivation und Fähigkeit der PatientIn, in diesem Rahmen zweckvoll mitzuarbeiten, die Übereinstimmung von PatientIn und TherapeutIn hinsichtlich der Ziele und Aufgaben, oder empathisches Verständnis und Involviertheit des TherapeutIn. Diese Variablen stellen die Basis dar, auf der die jeweils spezifischen technischen Interventionen wirken. In diesem Kontext wird die „gute“ Beziehung als notwendige jedoch nicht hinreichende therapeutische Prozessvariable definiert.

Zunehmend hat sich aber die Sichtweise durchgesetzt, dass sich - je nach Behandlungsansatz, Phase im Therapieverlauf, TherapeutInneneigenschaften (Beutler et al. 2004) und krankheitsbedingten PatientInnenmerkmalen - z. T. ganz unterschiedliche Qualitäten therapeutischer Beziehungen als günstig für Verlauf und Ergebnis erweisen (Biermann-Ratjen et al. 1995). Dieses erweiterte Konzept der Wirksamkeit der therapeutischen Beziehung erfordert eine stärker störungs- oder diagnosespezifische Erforschung der Therapiebeziehung, welche die Beziehungsregeln verschiedener PatientInnengruppen berücksichtigt. Dies wird in allen großen Therapieschulen, am stärksten in den interpersonellen Theorien und manchen gegenwärtigen psychoanalytischen Konzepten realisiert.

Eine ausschließlich ICD-basierte Behandlung (Berkin und Rief 2012) mit interaktionellem Schwerpunkt bietet daher die notwendige, jedoch nicht hinrei- chende Ausgangsbasis für die psychotherapeutische Behandlung von PatientInnen mit z. B. mangelnder oder/und schwankender Adhärenz/Compliance, mit aggressiv-manipulativer Tendenz, in oft chronisch krisenhafter selbst- oder fremdschädigender Einengung, mit Antriebslosigkeit oder misstrauischer Grundhaltung. Bei einer Vielzahl von Krankheitsbildern, welche die anhaltende Aktivierung von Ressourcen und motivierte Mitarbeit erfordert, kann dies von PatientInnen im Rahmen der üblichen psychologischen Gesprächsführung nicht genügend und nicht andauernd genug hergestellt werden.

Vielmehr bedarf es hier neben der atheoretisch beschreibenden ICD-Diagnostik einer erweiternden und theoriegeleitet-verstehenden psychotherapeutischen Diagnostik (vgl. Hochgerner 2020a, 2020b), welche die notwendigen Erklärungsmodelle zur Entstehung und Aufrechterhaltung einer Erkrankung als handlungsleitende Ausgangsbasis für klinischdiagnostisch begründete Interaktions- und Vorgangsweisen bietet. Psychotherapie schließt Kurzberatung, wie sie in Gestalt von psychologischer Behandlung angeboten wird, mit ein, geht jedoch in ihrer Kernkompetenz über Akutberatung und modularisierte Behandlungsschritte zur Erreichung eng umrissener Symptommilderung hinaus. Sie bietet hilfreiche, in jedem Einzelfall und jeder Behandlungsphase individuell an die PatientIn angepasste Behandlungskonzepte.

Ebensolche präzise angepassten Behandlungskonzepte benötigen für die genaue Indikationsstellung, für Prognose- und Risikoabschätzung (Leitner et al. 2014) eine umfassende Diagnostik und entsprechende diagnostische Kompetenzen.

\section{Die hohe Qualität der psychotherapeutischen Ausbildung}

Die psychotherapeutische Ausbildung, wie sie in Österreich auf der Grundlage des Psychotherapiegesetzes seit 1991 durchgeführt wird, hat im internationalen Vergleich eine besonders hohe Qualität, wie dies auch in der aktuellen internationalen „Spristad“-Studie bestätigt wird (Löffler-Stastka et al. 2019; Orlinsky et al. 2015).

$\mathrm{Zu}$ den zentralen Qualitätsmerkmalen dieser gesetzlich geregelten und qualitätsgesicherten Ausbildung zählen

- der Umfang der Ausbildung im Ausmaß von mindestens 250 bis 330 ECTS, abhängig vom methodenspezifischen Curriculum (BMG 2014; Hochgerner 2020c),

- der Aufbau auf einem Quellenberuf, der bei 70\% der eingetragenen PsychotherapeutInnen psychosozial ausgerichtet ist (Pawlowsky 2018); zugleich sind $73 \%$ der PsychotherapeutInnen akademisch ausgebildet (Pawlowsky 2020, 2021), 
- die klare wissenschaftstheoretische und handlungsleitende Verankerung in einem der vier psychotherapeutischen Cluster,

- die Ausbildung in mehreren Ausbildungsteilen (Theorieseminare und praktische Ausbildung im Propädeutikum und Fachspezifikum) mit einer durchschnittlichen berufsbegleitenden Ausbildungsdauer von aktuell 8,9 Jahren (Pawlowsky 2018),

- und die Orientierung an den vier gleichwertigen Elementen Theorie, Entwicklung therapeutischer Identität (Selbsterfahrung als reflexive und interaktionelle Kompetenz), Supervision und eigenständige Arbeit.

Die mehrfache und qualitätssichernde Selektion der psychotherapeutischen AusbildungskandidatInnen über alle Ausbildungsschritte hinweg unterscheidet sich weiters von den beiden anderen großen Zugängen zur Behandlung psychischer Erkrankungen (der ärztlichen Weiterbildung im Fach Psychiatrie und psychotherapeutische Medizin einerseits und der Weiterbildung in klinischer Psychologie andererseits):

- Auf Grund der Zäsur zwischen dem Propädeutikum und dem Fachspezifikum, der Aufnahmeverfahren in die fachspezifische Ausbildung, dem hohen Ausmaß an vorgeschriebener Selbsterfahrung und der engmaschigen, sich über mehrere Jahre erstreckenden Verschränkung von psychotherapeutischer Praxis und Supervision kommt es zu umfangreichen und mehrgliedrigen selbstselektiven Entscheidungsprozessen.

- Diese Entscheidungsprozesse kommen bereits früh zum Tragen: Aktuell vorliegenden Zahlen zufolge beenden ca. $25 \%$ der Personen, die das Propädeutikum begonnen haben, ihre Ausbildung nach dem ersten und allgemeinen Ausbildungsteil, dem Propädeutikum, ohne mit dem Fachspezifikum fortzusetzen (Pawlowsky 2018).

- Personen, die für eine fachspezifische Ausbildung in einer bestimmten Methode nicht geeignet erscheinen, werden nach komplexen Auswahlprozessen zur fachspezifischen Ausbildung nicht zugelassen oder auf die Ausbildung in einem anderen Psychotherapieverfahren verwiesen. Manche erkennen im Zuge der weiteren fachspezifischen Ausbildung durch Selbstkonfrontation in Praktika, in der Supervision und in der vorgeschriebenen Selbsterfahrung, dass die gewählte psychotherapeutische Methode für sie nicht geeignet ist.

- In diesem selektiven Auswahlprozess wird besonders die persönliche Eignung für ein bestimmtes anerkanntes Psychotherapieverfahren im Hinblick auf Persönlichkeit und fachspezifische Schwerpunktsetzungen untersucht. Dies hat zur Folge, dass die

- qualitative Eignung der Personen mit dem Abschluss einer psychotherapeutischen Ausbildung besonders hoch ist. $98 \%$ der fachspezifischen AusbildungskandidatInnen, die in eine fachspezifische
Ausbildung aufgenommen wurden, schließen die Psychotherapieausbildung positiv ab (Pawlowsky 2018).

Die für selbstselektive Entscheidungsprozesse erforderliche reflexive Kompetenz ist zudem ein zentraler psychotherapeutischer Wirkfaktor (die TherapeutInnenvariable erklärt 15-20\% der Ergebnisvarianz), mediiert die Interventionen (Taubner et al. 2015) und ist durch Selbsterfahrung und Supervision beeinflussbar (Nissen-Lie et al. 2013; Orlinsky et al. 2015). Sie ist aber auch durch Arbeits- und Umgebungsbedingungen veränderbar (Steinmair et al. 2020). Diese reflexive Kompetenz steht im Qualifikationsprofil und den damit verknüpften Lernzielen in der Psychotherapieaus- und Weiterbildung an prioritärer Stelle (European Association for Psychotherapy 2013). Auch die psychotherapeutische Medizin (als ärztliche Form psychotherapeutischer Kompetenz) hat im Vergleich dazu andere (z.T. und je nach Setting überlappende) Aufgaben und braucht dementsprechend andere Ausbildungswege.

Die Ausbildungsforschung in der Psychotherapie geht seit längerem der Frage nach, welche empirisch gestützten Faktoren die Entwicklung von PsychotherapeutInnen fördern (American Psychological Association 2006; Norcross 2002; Orlinsky et al. 2015). Wie die Ausbildung inhaltlich und didaktisch gestaltet werden kann und welche Kompetenzen sich abbilden, beschäftigten ForscherInnen in den letzten Jahren verstärkt (z. B. Duncan 2010; Kohl et al. 2009; Lorentzen et al. 2011; Orlinsky und Rønnestad 2005; Strauß und Kohl 2009; Sudak und Goldberg 2012).

Wie sekundäre Sozialisationsfaktoren die Entwicklung von Psychotherapeuten beeinflussen, ist hingegen wenig untersucht. In vielen Ländern - unter anderem auch in Deutschland und der Schweiz absolvieren Psychotherapie-AusbildungskandidatInnen vor der Therapieausbildung eine umfangreiche berufliche Ausbildung, entweder als PsychologInnen oder MedizinerInnen (vgl. dazu die Stichprobe in Lorentzen et al. 2011). Im Unterschied dazu ist der Zugang zur Psychotherapieausbildung in Österreich stark diversifiziert (vgl. auch Korunka und Hochgerner 2021). Ab 1991 wurden mit dem Psychotherapiegesetz (Kierein et al. 1991) aufgrund von Angebots-, Bedarfserhebungen und Entstigmatisierungsüberlegungen Schritte unternommen, Psychotherapie einer größeren Öffentlichkeit von PatientInnen anzubieten und einen niederschwelligeren Zugang zu schaffen. Damit ging einher, dass nicht nur ÄrztInnen, sondern auch Angehörigen anderer Berufsgruppen der Zugang zur Psychotherapieausbildung und in weiterer Folge auch zur Ausübung der Psychotherapie sowie bei gegebener Qualifikation auch zur Wahrnehmung lehrtherapeutischer Aufgaben gesetzlich gesichert bleiben sollte (Wißgott 2009). Diese Einbeziehung weiterer Berufe in die Ausbildung betrifft insbesondere PsychologInnen, PädagogInnen, Sozi- 
alarbeiterInnen, Pflegekräfte oder TheologInnen und somit Personengruppen, die auf Grund ihrer beruflichen Vorbildungen und Vorerfahrungen für eine Berufsausbildung zur PsychotherapeutIn potenziell qualifiziert sind und in Österreich schon vor dem Inkrafttreten des Psychotherapiegesetzes auf Grund ihrer human- und gesundheitswissenschaftlich orientierten Qualifikationen psychotherapeutisch ausgebildet worden waren (Springer-Kremser et al. 2002). Darüber hinaus können auch Personen, die einen anderen beruflichen Hintergrund aufweisen, bei besonderer, speziell auszuweisender Eignung nach einem Prüfverfahren zum Erlernen des PsychotherapeutInnenberufs zugelassen werden, um nach einer theoretischen und praktischen Ausbildung, die im Durchschnitt knapp neun Jahre lang dauert, ihre Kompetenzen und Erfahrungen in die psychotherapeutische Versorgung einbringen. Der Weg zum Erwerb ebendieser Kompetenzen ist in Tab. 1 dargestellt und mit ähnlichen Berufsausbildungen verglichen.

Löffler-Stastka et al. (2018) geben einen Überblick über die aktuelle Ausbildungssituation mit derzeit 23 in Österreich anerkannten Psychotherapieverfahren (Bundesministerium 2014) und 39 aktiven ausbildungsberechtigten Institutionen. Die in Österreich vertretenen Psychotherapiemethoden, die gelehrt werden, basieren nach ihrer Tradition, Schule und Methodik im Wesentlichen auf vier unterschiedlichen theoretischen, insbesondere wissenschaftstheoretischen, forschungsmethodologischen und anthropologischen Prämissen, die den oben erwähnten psychotherapeutischen Clustern entsprechen (PPT, VT, HPT, SPT). Aufgrund der strukturellen Besonderheit des Zugangs zur Psychotherapieausbildung bietet die Breite der österreichischen Psychotherapieschulen ein unverzichtbares Umfeld für die Beforschung der Relevanz des professionellen Basisberufs für die Entwicklung von PsychotherapeutInnen, deren Einstellungen und therapeutische Identitätsbildung. In einer länderübergreifenden Untersuchung zeigen sich Einflussfaktoren wie Haltung, Lebenszufriedenheit, ökonomische Verhältnisse und Stress auf die Entwicklung von PsychotherapeutInnen als wesentlich förderlich, ebenso die Diversität in der Ausbildung oder eine Inklusion von Minderheiten in die Ausbildung (Löffler-Stastka et al. 2018, 2019).

\section{Die Versorgungsleistung der Psycho- therapeutInnen}

Die Zahl der in die Berufsliste eingetragenen Personen mit derzeit 10.415 PsychotherapeutInnen (Stand 6/2020) verweist auf eine besonders hohe Motiviertheit des Berufsstandes, da die Ausbildung mit einer Länge von im Schnitt 8,9 Jahren - im Gegensatz zu den anderen gesetzlich anerkannten Heilberufen in keiner Weise staatlich finanziert ist. Zugleich verweist die durchschnittlich geringe Zahl an geleisteten Therapiestunden freiberuflicher PsychotherapeutIn- nen (Pawlowsky 2018; GÖG 2020) auf die auch nach 30 Jahren Psychotherapiegesetz nicht hinreichend eingelösten Aufgaben des Gesundheitssystems:

- Das vorhandene Angebot an kassenfinanzierten Plätzen für Psychotherapie stellt in Verbindung mit den geltenden Bezuschussungsregeln eine minimale Basisversorgung sicher. Darüber hinaus ist in allen Bundesländern ein weiterer Versorgungsbedarf gegeben (Grabenhofer-Eggerth und Sator 2019).

- Daraus folgt, dass ein gleicher und kassenfinanzierter Zugang für alle ÖsterreicherInnen zur Psychotherapie nach wie vor nicht gegeben ist. Der Nachfrage nach Psychotherapie als Sachleistung und dem Bedarf an Psychotherapie kann aufgrund der Kontingentierung des Angebots an kassenfinanzierten Psychotherapieplätzen nicht annähernd angemessen nachgekommen werden (Grabenhofer-Eggerth und Sator 2019). Dies schafft eine wesentliche und anhaltende Ungleichbehandlung psychisch Erkrankter gegenüber anderen Erkrankten im Versorgungssystem (Grabenhofer-Eggerth und Sator 2019).

- Eine gleiche und angemessene Abgeltung für die psychotherapeutischen Leistungen aller PsychotherapeutInnen im Gesundheitssystem ist nach wie vor inexistent. Die Umsetzung einer adäquaten und bereits in der 51. ASVG-Novelle (1993) versprochenen Integration psychotherapeutischer Dienste in ausreichender Stundenzahl in die stationäre Gesundheitsversorgung erfolgte noch nicht.

Die Benachteiligung psychisch Erkrankter wird in Österreich ohne Unterbrechung nach 1945 fortgeschrieben. Seit 2020 sind die häufigsten Erkrankungen der ÖsterreicherInnen psychische Erkrankungen (GÖG 2020). $\mathrm{Zu}$ den Aufgaben der Gesundheitsdienste gehört auch ein diversifiziertes Angebot an Leistungen, um dem Gegenstandsbereich der Psychotherapie, den unterschiedlichen Problemlagen von PatientInnen und der Subjektivität des Menschen im Sinne des $\S 1$ des Psychotherapiegesetzes gerecht zu werden.

Im Zuge der Weiterentwicklung des existierenden Versorgungssystems, das die erwähnten Mängel aufweist, bedarf es der Berücksichtigung des Umstandes, dass die reflexive Kompetenz von PsychotherapeutInnen (als bedeutsamen Faktor für die Wirksamkeit von Psychotherapie) nur dann zum Tragen kommen kann, wenn PsychotherapeutInnen eine angemessene Ausbildungs- und Arbeitsumgebung vorfinden, die von der öffentlichen Finanzierung von Psychotherapie mit abhängig ist. Darüber hinaus wird zu berücksichtigen sein, dass bereits PsychotherapeutInnen in Ausbildung unter Supervision in einem hohen Ausmaß Versorgungsleistungen erbringen: Unter Bezugnahme auf unterschiedliche Ausbildungsphasen bedarf es eines abgestuften Systems, in dem die Zulassung zur Ausübung psychotherapeutischer Tätigkeiten mit der öffentlichen Finanzierung von Psychotherapie und der 
Schaffung adäquater Ausbildungs- und Arbeitsumgebungen (etwa in Gestalt von psychotherapeutischen Lehr- und Versorgungsambulanzen) verschränkt wird.

\section{Psychotherapie ist eine eigenständige Disziplin}

Der historisch gleiche Entstehungszeitraum von naturwissenschaftlich beschreibender Psychiatrie, klinisch-messender Psychologie und verstehend-interaktioneller Psychotherapie mit dem vorwissenschaft- lich selben Untersuchungsgegenstand der Phänomene abweichenden psychischen Erlebens und Verhaltens zeigt einerseits die Verwobenheit der drei unterschiedlichen heilberuflichen Sichtweisen und zugleich die Deutungshoheit der Psychotherapie als eigene Form der Humanwissenschaft und eigenständiges Heilungsverfahren im interaktionellen Zugang zur leidenden Person.

Psychotherapie als Leitwissenschaft ist in der interaktionellen Heilung psychischer Störungen derzeit

Tab. 1 Zum Vergleich zwischen Psychotherapie, klinischer Psychologie und psychotherapeutischer Medizin. (Stundenangaben stellen die Mindestanforderung dar)

\begin{tabular}{|c|c|c|c|}
\hline & Psychotherapie & Psychotherapeutische Medizin & Klinische Psychologie \\
\hline $\begin{array}{l}\text { Ausbildung: Selbsterfah- } \\
\text { rung }\end{array}$ & $\begin{array}{l}\text { 250h 1. Abschnitt/Propädeutikum: } 50 \mathrm{~h} \text { und im } \\
\text { 2. Abschnitt/Fachspezifikum: } 200 \mathrm{~h} \text { schulen-/ } \\
\text { methodenspezifisch, 2-4 Jahre }\end{array}$ & $\begin{array}{l}\text { 150h schulen-/methodenspezifisch im Hauptfach, } \\
1-2 \text { Jahre }\end{array}$ & $76 \mathrm{~h}$ \\
\hline Ausbildung: Supervision & $\begin{array}{l}170 \mathrm{~h}: \\
20 \mathrm{~h} \text { Propädeutikum und } 150 \mathrm{~h} \text { Fachspezifikum } \\
\text { schulen-/methodenspezifisch }\end{array}$ & $\begin{array}{l}220 \mathrm{~h}: \\
\text { davon } 120 \mathrm{~h} \text { schulen-/methodenspezifisch }\end{array}$ & $120 \mathrm{~h}$ \\
\hline Ausbildung: Theorie & $\begin{array}{l}1065 \mathrm{~h} \\
\text { 1. Abschnitt/Propädeutikum } 765 \mathrm{~h} \text { methoden- } \\
\text { übergreifend und 2. Abschnitt Fachspezifikum: } \\
300 \mathrm{~h} \text { schulen-/methodenspezifisch }\end{array}$ & $600 \mathrm{~h}$ schulen-/methodenspezifisch & $340 \mathrm{~h}$ \\
\hline $\begin{array}{l}\text { Ausbildung: Gesamtum- } \\
\text { fang }\end{array}$ & $\begin{array}{l}\text { 3115h incl. Praktika in 8-9 Jahren nach einem } \\
\text { gesetzlichen Quellenberuf bzw. individueller } \\
\text { Eignungsprüfung }\end{array}$ & 1870h in 6 Jahren nach dem Medizinstudium & $\begin{array}{l}536 \mathrm{~h} \text { in 2-3 Jahren nach dem } \\
\text { Psychologiestudium }\end{array}$ \\
\hline Quellenberufe & $\begin{array}{l}\text { Abgeschlossener (meist akademischer) human- } \\
\text { wissenschaftlicher Quellenberuf entsprechend } \\
\text { dem Selbstverständnis der Psychotherapie }\end{array}$ & $\begin{array}{l}\text { Ärzte mit abgeschlossenem Lehrgang „Psycho- } \\
\text { somatische Medizin“, sowie FA Psychiatrie; } \\
\text { Voraussetzung: positive Beurteilung im Aufnah- } \\
\text { meverfahren }\end{array}$ & $\begin{array}{l}\text { Abgeschlossenes Psychologie- } \\
\text { studium }\end{array}$ \\
\hline $\begin{array}{l}\text { Selektion in der Ausbil- } \\
\text { dung als Qualitätssiche- } \\
\text { rungskriterium }\end{array}$ & $\begin{array}{l}\text { Insbes. Fremdselektionsmechanismen durch } \\
\text { Grundberuf und positive Beurteilung im Aufnah- } \\
\text { meverfahren in das Fachspezifikum. } \\
\text { Zusätzlich hohe Selbstselektionsmechanismen: } \\
\text { rund } 60 \% \text { der Beginnerlnnen der propädeuti- } \\
\text { schen Ausbildung schließen auch erfolgreich ein } \\
\text { Fachspezifikum ab }\end{array}$ & $\begin{array}{l}\text { Insbes. Fremdselektionsmechanismen durch } \\
\text { Grundberuf, positive Beurteilung im Aufnahmever- } \\
\text { fahren für psychotherapeutische Medizin }\end{array}$ & $\begin{array}{l}\text { Insbes. Fremdselektionsme- } \\
\text { chanismen durch Grundberuf }\end{array}$ \\
\hline Praxisumfang & $\begin{array}{l}600 \text { Behandlungsstunden unter Supervision plus } \\
1030 \mathrm{~h} \text { Praktika: } \\
\text { 1. Abschnitt: } 480 \mathrm{~h} \\
\text { 2. Abschnitt: } 550 \mathrm{~h} \text { (mind. } 150 \mathrm{~h} \text { im klinischen } \\
\text { Bereich) }\end{array}$ & $\begin{array}{l}600 \mathrm{~h} \text { Ärztliche Tätigkeit unter psychotherapeu- } \\
\text { tischen Gesichtspunkten + weitere } 600 \text { Behand- } \\
\text { lungsstunden methodenspezifisch (mind. } 6 \text { Fälle, } \\
\text { davon mind. } 3 \text { über } 40 \mathrm{~h} \text { ) }\end{array}$ & $\begin{array}{l}\text { Praxiszeit } 2098 \mathrm{~h} \text {, keine defi- } \\
\text { nierten Behandlungsstunden }\end{array}$ \\
\hline Diagnostik & $\begin{array}{l}\text { Grundzüge der psychiatrischen und psycholo- } \\
\text { gischen Diagnostik, zusätzlich behandlungs- } \\
\text { relevante und schulen-/methodenspezifische } \\
\text { psychotherapeutische Diagnostik (inkl. Bezie- } \\
\text { hungs- und Prozessdiagnostik) }\end{array}$ & $\begin{array}{l}\text { Psychiatrische Diagnostik, integrativ entlang } \\
\text { des somato-, sozio- und psychotherapeutischen } \\
\text { Ansatzes }\end{array}$ & $\begin{array}{l}\text { Psychologische Diagnostik } \\
\text { (mit einem besonderen Fokus } \\
\text { auf klinisch-psychologische } \\
\text { Diagnostik) und Grundzüge der } \\
\text { psychiatrischen Diagnostik }\end{array}$ \\
\hline Theoretische Grundlagen & $\begin{array}{l}\text { Klare wissenschaftstheoretische Verankerung } \\
\text { in einem Menschenbild und in der jeweiligen } \\
\text { Psychotherapietheorie }\end{array}$ & $\begin{array}{l}\text { Methodenspezifisch (Hauptfach } 150 \mathrm{~h} \text { eine Tradi- } \\
\text { tion, Zusatzfach } 40 \mathrm{~h} \text { andere Tradition, } 2 \text { Ergän- } \\
\text { zungsfächer je } 20 \mathrm{~h} \text { ) }\end{array}$ & $\begin{array}{l}\text { Eklektisch auf der Grundlage } \\
\text { verschiedenster psychologi- } \\
\text { scher Theorien }\end{array}$ \\
\hline Behandlungskonzepte & $\begin{array}{l}\text { Fundiert methodenspezifisch und evidenzbasiert } \\
\text { Prozess- und beziehungs-orientiert }\end{array}$ & $\begin{array}{l}\text { Methodenspezifisch mit dem Schwerpunkt eines } \\
\text { Hauptfaches; Integration von Zusatzfach; } \\
2 \text { Ergänzungsfächer; alle entsprechend den } 4 \\
\text { grundlegenden psychotherapeutischen Tradi- } \\
\text { tionen, aber in verschiedenen Settingvarianten } \\
\text { evidenzbasiert }\end{array}$ & $\begin{array}{l}\text { Eklektisch und evidenzbasiert } \\
\text { In der Regel interventionsba- } \\
\text { siert }\end{array}$ \\
\hline Breite der Behandlung & Breit & Breit & Spezifisch \\
\hline $\begin{array}{l}\text { Zielgruppen der Behand- } \\
\text { lung }\end{array}$ & $\begin{array}{l}\text { Alle klinischen Störungsbilder, Persönlichkeits- } \\
\text { entwicklung, etc. }\end{array}$ & $\begin{array}{l}\text { Alle Psychiatrischen und Psychosomatischen } \\
\text { Störungsbilder }\end{array}$ & $\begin{array}{l}\text { Spezifische Zielgruppen, z. B. } \\
\text { Neuropsychologie, Gerontopsy- } \\
\text { chologie, Teilleistungsstörun- } \\
\text { gen etc. }\end{array}$ \\
\hline Klinische Evidenz & $\begin{array}{l}\text { Hoch für die vier therapeutischen Cluster auf der } \\
\text { Basis spezifischer und allgemeiner Wirkfaktoren }\end{array}$ & Nach den Kriterien der Evidenz-Basierten-Medizin & $\begin{array}{l}\text { Meist spezifisch interventi- } \\
\text { onsbasiert; in der Regel nicht } \\
\text { auf der Basis der allgemeinen } \\
\text { Wirkfaktoren }\end{array}$ \\
\hline
\end{tabular}


in den oben genannten vier Grundströmungen repräsentiert - ein weiteres Merkmal und Spezifikum der Psychotherapie. Sie wird damit in ihrer Vielfalt an $\mathrm{Zu}$ gängen der immer weiter zunehmenden Komplexität und Diversität menschlichen Erlebens und Verhaltens hinreichend gerecht: Sie reagiert in komplexer und adäquater Weise unter Einbeziehung sozialer, ökonomischer und ökologischer Momente auf individuelle und gesellschaftliche Entwicklungen und hat damit eine nachhaltige Bedeutung, die weit über die Heilbehandlung einzelner Symptombilder hinausgeht. Dies kommt auch darin zum Ausdruck, dass Psychotherapie als Disziplin die Befassung mit diesen Momenten in ihre Theoriebildungen miteinbezieht (vgl. Kadi et al. 2012).

Zeichnete sich in den 1990er-Jahren deutlich ab, dass sich Psychotherapie auf dem Weg zu einer eigenständigen Disziplin befindet, so ist zweieinhalb Jahrzehnte später festzuhalten, dass Psychotherapie inzwischen wesentliche Merkmale einer eigenständigen Disziplin nach Datler und Felt (1996) erfüllt:

Innerhalb eines langjährigen international existierenden Netzwerkes wird unter Einsatz von wissenschaftlich anerkannten Methoden permanent an einem breiten Spektrum an Themen gearbeitet, in deren Zentrum die psychotherapeutische Praxis und damit verbundene Theorien stehen (vgl. Wampold und Imel 2015).

Diese Theorien handeln insbesondere von

- der Theorie des psychotherapeutischen Prozesses,

- den Indikationen zur Psychotherapie,

- von psychotherapeutischen Wirkfaktoren und dem „Outcome“ psychotherapeutischer Praxis,

- von den Entstehungsbedingungen und Entstehungsprozessen der Problem- und Leidenszustände, die zur Indikation von Psychotherapie führen, mit besonderen Akzenten auf die Theorie der Spezifität und Genese psychischer Erkrankungen, ihrer Behandlung und ihrer Prophylaxe,

- von anthropologischen, persönlichkeits-, interaktions- und sozialisationstheoretischen Grundlagen,

- von professionstheoretischen Themen, die auch Fragen der psychotherapeutischen Aus-, Fort- und Weiterbildung betreffen,

- vom Spektrum wissenschaftlicher Methoden, die in der Psychotherapieforschung zum Einsatz kommen,

- sowie von der Beziehung zwischen der Disziplin der Psychotherapie und anderen wissenschaftlichen Disziplinen wie Medizin, Psychologie, Erziehungsund Bildungswissenschaft, Soziologie, Philosophie oder Theologie.

Innerhalb der Disziplin der Psychotherapie existieren

- gemeinsam geteilte Wissensbestände, die in Lexika, Handbüchern und Lehrbüchern dargestellt sind, die zum Teil clusterspezifisch, zum Teil aber auch clusterübergreifend konzipiert sind (z.B. Stumm und Pritz 2007; Slunetzko 2009; Fischer 2011; Lambert 2013),

- Kommunikationsstrukturen, zu denen insbesondere wissenschaftliche Journale, langjährig tätige wissenschaftliche Institute und Vereinigungen sowie regelmäßig stattfindende Kongresse, Tagungen und Symposien zählen,

- Selektions- und Reproduktionsprozesse (Ausbildungsprozesse), für die primär LehrtherapeutInnen und somit VertreterInnen der Disziplinen Verantwortung tragen (vgl. LehrtherapeutInnen-Richtlinie 2011);

- wissenschaftliche Diskurse, die der Selbstreflexion der Disziplin dienen und insbesondere von den Besonderheiten der Disziplin und ihrer Geschichte handeln (vgl. Norcross et al. 2011; Schmidbauer 2012).

In der Disziplin der Psychotherapie gelten Werte und Normen des wissenschaftlichen Arbeitens, die auch in anderen Disziplinen als verbindlich angesehen werden (Felt et al. 1995). Und PsychotherapeutInnen werden inzwischen nahezu durchgängig als ExpertInnen für das Wissen um Psychotherapie, für die Weiterentwicklung der Disziplin und für die gesellschaftlich relevante Nutzung des psychotherapeutischen Wissens insbesondere in den Bereichen der psychotherapeutischen Praxis (Behandlung), Versorgung und Prophylaxe begriffen.

\section{Die Binnenstrukturen der Disziplin der Psychotherapie}

Die Disziplin der Psychotherapie weist in mehrfacher Hinsicht Binnenstrukturen auf. Im Bereich der Psychotherapieforschung existieren etwa Strukturen der qualitativen und quantitativen Psychotherapieforschung in Verbindung mit dem Einsatz von Mixed Methods in Korrespondenz mit Konzeptforschung, Einzelfallforschung und Untersuchungen mit großen Stichproben (Fischer 2011, S. 107 ff.).

Eine andere Binnenstruktur verdeutlicht unterschiedliche Strömungen der Psychotherapie, die den bereits mehrfach genannten vier Clustern zugeordnet werden können (PPT, VT, HPT, SPT). All diesen Strömungen ist die Auffassung gemeinsam, dass es in psychotherapeutischen Prozessen nötig ist, auch zu den tieferen, persönlichkeitsbestimmenden Schichten der Problemlagen von psychotherapiebedürftigen Menschen zu kommen, die erst dann erfasst und hilfreich bearbeitet werden können, wenn sie innerhalb der therapeutischen Beziehung spürbar, erkannt und verstanden werden. Deshalb zeichnet sich die psychotherapeutische Ausbildung durch den hohen Anteil an psychotherapeutischer Eigenerfahrung als Voraussetzung für diese unverzichtbare Dimension des psychotherapeutischen Arbeitens aus.

Obgleich dieser Aspekt innerhalb der psychotherapeutischen Cluster etwas unterschiedlich gewichtet 
wird, unterscheidet sich die psychotherapeutische Ausbildung in dieser qualitativ unverzichtbaren Dimension in allen Clustern von den Ausbildungsprozessen in angrenzenden Disziplinen. Dort wird diesen Aspekten in deutlich geringerem Ausmaß und nur situativ Rechnung getragen, weshalb in dieser Hinsicht auch andere Anforderungen an ärztliche oder klinisch-psychologische Ausbildungen und Praxisformen zu stellen sind:

Die therapeutische Begegnung bildet als interaktionelle Situation den Rahmen zur Analyse und heilender Bearbeitung leidvollen Erlebens und Verhaltens und steht damit im Zentrum jeder Psychotherapie. Damit wandelt sich auch die Aufgabe der Diagnostik krankheitswertiger Zustandsbilder: Psychotherapeutische Diagnostik steht als eigenständige und intersubjektiv begründete verstehende Beziehungsdiagnostik neben der psychiatrisch-erklärenden und psychologisch-experimentell messenden Diagnostik als jeweils nosologisch-kategoriale Formen der Krankheitsfeststellung (Hochgerner 2020a, 2020b; Höfner und Hochgerner 2020). Sie ermöglicht grundsätzlich und aktuell im psychotherapeutischen Prozess Auskunft über mögliche Entstehungszusammenhänge und Formen der Aufrechterhaltung leidvoller Symptomatik und setzt diese in Relation zur Persönlichkeit. Diagnostik wird in dieser Weise, begleitend zum therapeutischen Prozess, integraler, kontinuierlicher Bestandteil der Psychotherapie und ist in der Indikationsstellung zur Psychotherapie als prozessuale „Theragnostik“ ein qualitativ wesentlich vertiefender Zugang zum psychischen Erleben und Verhalten. Diagnostik geht damit weit über die Erfassung und $\mathrm{Zu}-$ schreibung von Krankheitsbildern, wie sie in gängigen psychiatrischen oder klinisch-psychologischen Systematiken gelistet und beschrieben werden, hinaus: Sie ist handlungsleitend in der Entwicklung und Zielfindung hilfreicher therapeutischer Haltungen und individualisierter Vorgangsweisen, fördert wesentlich die Motivation zur Mitarbeit von PatientInnen und ist somit für die Arbeitsbündnisse innerhalb der jeweils spezifischen therapeutischen Beziehung wesentlich. Deshalb beginnen sich psychotherapeutische Prozesse nicht erst nach der psychotherapeutischen Diagnostik zu entwickeln, sondern bereits mit dem Erstkontakt und dem Einsetzen psychotherapeutischdiagnostischer Aktivitäten, denen im oben erwähnten Sinn ein „theragnostischer“ Anspruch inhärent ist.

\section{Psychotherapie und ihre Beziehung zu Nachbardisziplinen}

Wissenschaftliche Disziplinen haben ihre Wurzeln zumeist in anderen Disziplinen. Prozesse der Herauslösung aus diesen Disziplinen und Prozesse der Differenzierung von diesen Disziplinen führen dazu, dass eigenständige Disziplinen entstehen.

Die Wurzeln der Psychotherapie sind in diesem Sinn in verschiedenen Disziplinen, in wesentlichen
Punkten aber auch in Diskursen zu finden, die außerhalb dieser Disziplinen geführt wurden bzw. spezifischen Disziplinen nicht eindeutig zugeordnet werden können. Ein markantes Beispiel geben dafür die Anfänge der Psychoanalyse $\mathrm{ab}$, aus der heraus sich das Cluster der psychoanalytisch-psychodynamischen Psychotherapie (PPT) entwickelte:

Sigmund Freud, der Begründer der Psychoanalyse, war habilitierter Mediziner und kam als solcher mit den Vorläufern und Anfängen der Psychotherapie insbesondere in Wien und in Frankreich in den 1880er-Jahren in Berührung. Als wissenschaftlich interessierter und wissenschaftlich tätiger Arzt leistete er mit der Begründung der Psychoanalyse Bahnbrechendes auf dem Gebiet der Psychotherapie. Die Entstehung der Psychoanalyse war aber nur zum Teil auf die wissenschaftliche Befassung mit Psychotherapie innerhalb der Medizin zurückzuführen. Von größerem Gewicht war vielmehr der Umstand, dass sich zunächst in Wien und bald darauf international ein Netzwerk von wissenschaftlich arbeitenden PsychoanalytikerInnen etablierte, das außerhalb der universitär verankerten Disziplinen wie Medizin, Psychologie oder Pädagogik existierte.

Für die Etablierung und Verbreitung der Psychoanalyse war es zwar nicht unerheblich, dass diesem psychoanalytischen Netzwerk auch MedizinerInnen sowie PsychologInnen, PädagogInnen, TheologInnen und VertreterInnen verschiedener anderer Geisteswissenschaften angehörten, die zum Teil in die akademischen Strukturen dieser Disziplinen (etwa als ProfessorInnen oder AssistentInnen) eingebunden waren. Die Orte, an denen psychoanalytisch-psychotherapeutische Praxis geleistet wurde, und die Institutionen, in denen die wissenschaftliche Diskurse über psychoanalytische Theorien und Konzepte geführt wurden, lagen zumeist aber außerhalb universitärer Strukturen und konnten den bestehenden Disziplinen auch gar nicht eindeutig zugeordnet werden, zumal die Entstehung und Verbreitung der Psychoanalyse von maßgeblichen VertreterInnen der seinerzeit etablierten Disziplinen kritisch gesehen und zum Teil sogar bekämpft wurden. Historischen Analysen ist zu entnehmen, wie vielschichtig und spannungsreich sich von Beginn an das Verhältnis zwischen der Psychoanalyse und jenen Disziplinen dargestellt hat, die heute zu den wichtigsten psychotherapeutischen Nachbardisziplinen zählen (siehe etwa zum Verhältnis zur Medizin Böker 2006; Hierdeis und Scherer 2018; zur Psychologie Elliger 1986; Nitzschke 1989; zur akademischen Pädagogik Wininger 2011; oder zu Philosophie, Theologie und Soziologie Cremerius 1981; Scheidt 1986).

Auch das Wirken vieler anderer - aus unterschiedlichen Disziplinen kommenden - historischer Persönlichkeiten, die der Entwicklung der Psychotherapie wesentliche Impulse gaben, kann man ablesen, dass Psychotherapie nicht als Teilgebiet einer Disziplin, 
die bereits seit längerer Zeit besteht, begriffen werden kann:

Alfred Adler war Arzt und bis 1911 Mitglied in Freuds multidisziplinär zusammengesetzten Mittwoch-Gesellschaft, ehe er sich von Freud trennte und die Individualpsychologie begründete, die nicht zuletzt dadurch weite Verbreitung fand, dass sie im „Roten Wien“ in pädagogischen Institutionen gelehrt und auch mit therapeutischem Anspruch praktiziert wurde.

Jakob L. Moreno, der Philosophie und Medizin studiert hatte, entfaltete in den 1920er-Jahren den interaktionell-begegnungsorientierten, sozialwissenschaftlich begründeten Ansatz der humanistischen Psychotherapie, der Psychotherapie in Gruppen etablierte und wesentlichen Einfluss auf die amerikanische Sozialpsychologie, Pädagogik und Beratungskultur („Counseling“) hatte.

Victor Frankl entwickelte nach seinen Studien der Medizin und Philosophie ab den 1920er-Jahren seinen Logotherapeutischen Therapieansatz, der als „3. Wiener Schule der Tiefenpsychologie“ gilt.

Carl Rogers begründete in den 1940er-Jahren in den USA den personzentrierten Ansatz und damit eine zentrale Schule der humanistischen Psychotherapie, die sich unter anderem auch als kritische Gegenposition zur damaligen akademischen Psychologie verstand.

Albert Ellis und Aaron Beck entwickelten als Repräsentanten der Psychologie und Psychiatrie von den 1950er-Jahren an den Ansatz der Rational-Emotiven Psychotherapie und trugen damit wesentlich zur „kognitiven Wende“ in der Verhaltenstherapie bei.

Virginia Satyr entwickelte ab den 1950er-Jahren als Sozialarbeiterin in der Familienhilfe mit der Begründung der „Familientherapie“ spezielle Formen des Zugangs zur Mehrpersonenpsychotherapie in sozialen Netzwerken.

Dass PsychotherapeutInnen seit den Anfängen der modernen Psychotherapie mit Medizin und Psychologie, darüber hinaus aber auch mit weiteren Disziplinen der Geistes-, Human- und Sozialwissenschaften samt den damit verbundenen Praxisfeldern vertraut waren, hatte zur Folge, dass fachlich breit gefächerte Expertisen in das Feld der Psychotherapie eingebracht wurden. Psychotherapie entspricht damit der bio-psycho-sozialen Realität des Individuums und dem mehrperspektivischen Krankheitsbegriff der WHO besser als vorrangig naturwissenschaftlich respektive einheitswissenschaftlich orientierte Zugänge zum leidenden Menschen. Umgekehrt flossen psychotherapeutische Erkenntnisse auch in die Diskurse anderer Disziplinen (siehe oben) und in die damit verbundenen Praxisfelder zurück, einschließlich jener Felder, die für Sozialkritik und Sozialreformen von großer Bedeutung sind.

Diese Anregungs- und Austauschprozesse gewinnen mit der Etablierung der Psychotherapie als Disziplin an Intensität und Qualität und finden ihre in- stitutionalisierte Verankerung überall dort, wo die gesetzliche Regelung der psychotherapeutischen Ausund Weiterbildung sicherstellt, dass das plurale Verhältnis der Psychotherapie auf hohem Wissenschaftsund Ausbildungsniveau zu anderen Disziplinen befördert und weiterentwickelt wird. Dies verhindert Begehrlichkeiten, durch Umformung und letztlich Zerstörung der Psychotherapie als umfassende und eigenständige Disziplin und Wissenschaft einzelne konstituierende Merkmale aus dem zentralen Wirkzusammenhang zu reißen und bloß als Teildisziplin anderer Gesundheitsberufe auszuweisen. Die vielschichtige und zunehmende forschungs-, heilungsund gesellschaftliche Relevanz der Psychotherapie wäre dann durch die Beförderung von Eindimensionalität minimiert.

\section{Conclusion}

Aus der Beschäftigung mit der aktuellen Literatur aus der Psychotherapieforschung (Lambert 2013) können folgende Thesen abgeleitet werden:

Psychotherapie - Unverzichtbar für die Behandlung psychischer Erkrankungen

\section{Psychotherapie ist evidenzbasiert und effizient}

- Psychotherapie hat eine hohe und nachhaltige Behandlungswirksamkeit,

- ist durch die empirische Psychotherapieforschung für alle vier in Österreich anerkannten Ausrichtungen (Methodencluster) wirksam ausgewiesen und

- ihr Erfolg ist einer psychopharmakologischen Behandlung vergleichbar.

- Durch die langfristige Behandlungswirkung folgt eine

- beachtenswerte Kosteneffizienz, die eine

- nachhaltige Senkung der Gesundheitskosten bedeutet.

- PsychotherapeutInnen entwickeln für jede/n PatientIn einen notwendigen und eigenen Zugang und individuell hilfreichen Therapieplan.

Kernbereiche der Psychotherapie

Im Fokus der Psychotherapie steht die Subjektivität des individuellen Erlebens von PatientInnen, in denen die psychischen und psychosomatischen Symptome, Krankheits- und Leidenszustände von PatientInnen gründen.

\section{Psychotherapie}

- Die Psychotherapie greift das subjektive Leiden und/oder die Störung der/s PatientIn auf und bearbeitet umfassend und prozessorientiert alle Bedeutungen und Aspekte der jeweiligen Problematik und ist ressourcenorientiert.

- Psychotherapie beruht auf einer klaren theoretischen Verankerung und einer Haltung (einem Men- 
schenbild) der/s PsychotherapeutIn, die in einer theoretischen und praktischen Ausbildung zur Entwicklung einer psychotherapeutischen Identität erworben wird.

- Im zeitlichen Vergleich ist die Ausbildung umfangreich (Zugangsberuf/Studium plus mindestens 3115h über 8-9 Jahre verteilt).

- Zahlreiche fremd- und selbstselektive Schritte während der Ausbildung erhöhen die Qualität.

- Sie wird international in 4 anerkannten Methodenclustern psychodynamischer, humanistischer, systemischer oder verhaltenstherapeutischer Ausrichtung angeboten.

- In der konkreten Umsetzung kann das in psychotherapeutischer Krisenintervention, in Kurz- oder Langzeittherapie in verschiedenen Settings (Einzel-, Gruppen-, Familientherapie etc.) erfolgen.

- Die Psychotherapie stellt ein professionelles und umfassendes Behandlungskonzept dar. Das Behandlungskonzept ist umfassend und wirksam für alle klinischen Störungsbilder.

- Die Wirksamkeit der Psychotherapie ergibt sich im Zusammenwirken aus der therapeutischen Beziehung (30\%), spezifischen Interventionen (10\%), TherapeutInnenfaktoren (20\%) und der PatientInnencharakteristika ( $40 \%)$.

\section{Psychotherapeutische Diagnostik}

- Psychotherapeutische Diagnostik erfasst den aktuellen Leidensdruck und die vorliegende krankheitswertige Symptomatik.

- Sie gewinnt darüber hinaus jedoch aus lebensgeschichtlichen und aktuellen Daten klinisches Wissen über Entstehung und Aufrechterhaltung von leidvollem Erleben und Verhalten.

- Damit entsteht ein Persönlichkeitsprofil, das es erlaubt, die für diese Person passende hilfreiche therapeutische Haltung und Vorgangsweise individuell und theoriegeleitet für jeden therapeutischen Schritt zu entwickeln.

- Körperliches, Soziales und Psychisches Erleben und Verhalten werden in der psychotherapeutischen Diagnostik in ihrer subjektiven Bedeutung und Wechselwirkung permanent erfasst und hilfreich verknüpft.

- Psychotherapeutische Diagnostik ist nicht nur die Basis und Ausgangspunkt von Behandlung, sondern zugleich und wesentlich auch kontinuierlicher Bestandteil jeder therapeutischen Einheit, die das subjektive Erleben und Verhalten zum Zentrum der Behandlung macht.

- Damit wird in der diagnostisch geleiteten hilfreichen psychotherapeutischen Beziehung die Aktivierung der jeweils eigenen Möglichkeiten und Ressourcen zur Krankheitsbewältigung ermöglicht und als nachhaltig hilfreiche Persönlichkeitsveränderungen etabliert.
Geht man in weiterer Konsequenz der Frage nach, ob es sich um eine eigenständige Wissenschaftsdisziplin handelt, muss dies aus dem derzeitigen Stand des Wissens (Wampold und Imel 2015) verneint werden: im Sinne Kuhns müssten alle Psychotherapieforschungsergebnisse inkommensurabel $\mathrm{zu}$ den bisherigen Forschungsergebnissen aus Medizin, Psychologie, Neurobiologie, Evolutionslehre, Entwicklungspsychologie, Bildungswissenschaften, etc. sein, wenngleich kleinere wissenschaftliche Revolutionen zu beobachten waren (kognitive Wende, mixed-methods Forschungsdesigns, etc.). Stellt man die PatientIn partizipativ in den Mittelpunkt (Krause et al. 2020), ist für die Versorgung psychisch kranker Menschen die Beibehaltung der Vielgestaltigkeit unter Berücksichtigung der Unterschiede unterschiedlicher Disziplinen, Professionen und der evidenzbasierten Praxis von zentraler Bedeutung. Je nach Blickwinkel wird der Aspekt der Disziplin, des Berufes oder der Forschungsmethodologie in den weiteren Diskussionen zur wissenschaftlichen Weiterentwicklung der Psychotherapie unterschiedliche Betonung finden, um dadurch auch einem metatheoretischen Diskurs gerecht $\mathrm{zu}$ werden - dies jedoch vorrangig zum Wohle der/s einzelnen PatientIn.

Funding Open access funding provided by Medical University of Vienna.

Interessenkonflikt W. Datler, M. Hochgerner, C. Korunka, H. Löffler-Stastka und G. Pawlowsky geben an, dass kein Interessenkonflikt besteht.

Open Access Dieser Artikel wird unter der Creative Commons Namensnennung 4.0 International Lizenz veröffentlicht, welche die Nutzung, Vervielfältigung, Bearbeitung, Verbreitung und Wiedergabe in jeglichem Medium und Format erlaubt, sofern Sie den/die ursprünglichen Autor(en) und die Quelle ordnungsgemäß nennen, einen Link zur Creative Commons Lizenz beifügen und angeben, ob Änderungen vorgenommen wurden.

Die in diesem Artikel enthaltenen Bilder und sonstiges Drittmaterial unterliegen ebenfalls der genannten Creative Commons Lizenz, sofern sich aus der Abbildungslegende nichts anderes ergibt. Sofern das betreffende Material nicht unter der genannten Creative Commons Lizenz steht und die betreffende Handlung nicht nach gesetzlichen Vorschriften erlaubt ist, ist für die oben aufgeführten Weiterverwendungen des Materials die Einwilligung des jeweiligen Rechteinhabers einzuholen.

Weitere Details zur Lizenz entnehmen Sie bitte der Lizenzinformation auf http://creativecommons.org/licenses/by/4. $0 /$ deed.de.

\section{Literatur}

Ablon, J. S., \& Jones, E. E. (2005). On analytic process. Journal of the American Psychoanalytic Association, 53, 541-568.

Ablon, S., \& Jones, E. E. (1998). How expert clinicians' prototypes of an ideal treatment correlate with outcome in psychodynamic and cognitive-behavioral therapy. Psychotherapy Research, 8, 71-83. 
American Psychological Association (2006). Evidencebased practice in psychology. American Psychologist, 61,271-285.

Berkin, M., \&Rief, W. (2012). Klinische Psychologie und Psychotherapie für Bachelor. Berlin: Springer.

Beutler, L.E., Malik, M., Alimohamend, S., Harwood, T.M., Talebi,H., Noble, S., \&Wong, E. (2004). Therapist variables. In M. J. Lambert (Hrsg.), Bergin and Garfield's Handbook of Psychotherapy and Behavior Change(Bd.5,S.227-306). New York: Wiley.

Biermann-Ratjen, E.M., Eckert, J., \& Schwartz, H.J. (1995). Gesprächspsychotherapie. Verändern durch Verstehen. Stuttgart: Kohlhammer.

Blatt, S. J. (1995). Representational structures in psychopathology. In D. Cicchetti \& S. L. Toth (Hrsg.), Rochester symposium on developmental psychopathology. Emotion, cognition, and representation (Bd. 6, S. 1-33). Rochester: University of Rochester Press.

Blatt, J., \& Shichman, S. (1983). Two primary configurations of psychopathology. Psychoanalysis and Contemporary Thought, 6(2), 187-254.

Böker, H. (Hrsg.). (2006). Psychoanalyse und Psychiatrie. Geschichte, Krankheitsmodelle und Therapiepraxis. New York: Springer.

Brittlebank, A., Hermans, M., Bhurga, D., Pinto da Costa, M., Rojnic-Kuzmann, M., Fiorillo, A., Kurimay, T., Hanon, C., Wassermann, D., \& van der Gaag, R. J. (2016). Training in psychiatry throughout Europe. Eur Arch Psychiatry Clin Neurosci, 266, 155-164.

Bundesgesetz über die Führung der Bezeichnung „Psychologin“ oder „Psychologe“ und über die Ausübung der Gesundheitspsychologie und der Klinischen Psychologie (Psychologengesetz 2013). StF: BGBl. I Nr. 182/2013 (NR: GP XXIV IA 2360/A AB 2572 S. 213. BR: 9041 AB 9067 S. 823.) [CELEX-Nr.: 32011L0024, 32011L0095]. Zugegriffen: 11. Februar 2021.

BGBl. Nr. 335/1993 - BUNDESGESETZBLATT 335. Bundesgesetz, mit dem das Allgemeine. Sozialversicherungsgesetz (51. Novelle zum. ASVG), das Beamten-Kranken- und Unfallversicherungsgesetz (...) getroffen werden. https:// ris.bka.gv.at, 1993_337_0 > 1993_337_0. Zugegriffen: 11. Februar 2021.

BMSGPK (2011/2020). LehrtherapeutInnen-Richtlinie für das Fachspezifikum. (Richtlinie des Gesundheitsressorts auf Grundlage eines Gutachtens des Psychotherapiebeirats. Psychotherapie Forum, 9, Suppl. 2(2/2011), 46-47, ergänzt und aktualisiert am 05.08.2010. Originaltext: www. sozialministerium.at(PDF-Datei).Zugegriffen:11.Februar 2021.

Bundesministerium (2014). Anerkannte Psychotherapiemethoden in Österreich: Patientinnen/Patienten-Information über die in Österreich anerkannten psychotherapeutischen Methoden (Stand 2014). https://www. bmgf.gv.at/home/Gesundheit/Psychische_Gesundheit/ In_Oesterreich anerkannte Psychotherapiemethoden. Zugegriffen: 10. Sept. 2018. Veröffentlicht vom österreichischen Bundesministerium für Arbeit, Soziales, Gesundheitund Konsumentenschutz (BMASGK) (2014).

Bundesministerium (2019). Gesundheitsberufe in Österreich. Herausgegeben vom österreichischen Bundesministerium für Arbeit, Soziales, Gesundheit und Konsumentenschutz. file:///C:/Users/admin/Downloads/ gesundheitsberufe_2019,_mai_2019.pdf.Zugegriffen: 15. Juni 2020.

Bundesministerium für Gesundheit (2014). Leitfaden zur Bewertung der Psychotherapieausbildung mit ECTS für Propädeutika und Fachspezifika; Ausschuss für fachspe- zifische Angelegenheiten; Psychotherapiebeirat. https:// www.sozialministerium.at/Themen/Gesundheit/Medi zin-und-Gesundheitsberufe/Berufe-A-bis-Z/Psycho therapeutin,-Psychotherapeut/ECTS-Bewertungpsychotherapeutischer-Pro\%C3\%A4deutika-und-Fach spezifika.html.Zugegriffen: 17. Juni 2020.

Butler, S.F., \& Strupp, H.H. (1986). Specific and nonspecific factors in psychotherapy: a problematic paradigm for psychotherapy research. Psychotherapy: Theory, Research, and Practice, 23, 30-40.

Cain, D. J. (2016). Humanistic psychotherapies. Handbook of research and practice. Washington, DC:APAPress.

Cooper, M., Watson, J.C., \& Hölldampf, D. (2013). Personcentered and experiential therapies work. Ross-on-Wye, United Kingdom: PCCS books.

Cremerius, J. (Hrsg.). (1981). Die Rezeption der Psychoanalyse in der Soziologie, Psychologie und Theologie im deutschsprachigen Raum bis 1940. Frankfurt:Suhrkamp.

Cuijpers, P. (1998). Minimising interventions in the treatment and prevention of depression: taking the consequences of the "dodo bird verdict. Journal of Mental Health, 7, 355-365.

Cuijpers, P., van Straten, A., Wamerdam, L., \& Andersson, G. (2008b). Psychological treatment of depression: a metaanalytic database of randomized studies. BMC Psychiatry, $8(1), 26$.

Cuijpers, P., van Straten, A., Warmerdam, L., \&Smits, N. (2008a). Characteristics of effective psychological treatments of depression: a metaregression analysis. Psychotherapy Research, 18, 225-236.

Cuijpers, P., Noma, H., Karyotaki, E., Vinkers, C.H., Cipriani, A., \& Furukawa, T.A. (2020). A network meta-analysis of the effects of psychotherapies, pharmacotherapies and their combination in the treatment of adult depression. World Psychiatry, 19, 92-107. https://doi.org/10. 1002/wps.20701.

Datler, W., \& Felt, U. (1996). Psychotherapie-eine eigenständigeDisziplin? In A. Pritz (Hrsg.), Psychotherapie-eine neue Wissenschaft vom Menschen (S. 45-73). Wien: Springer.

Datler, W., Drossos, A., Korunka, Ch , \& Gornik, E. (Hrsg.). (2021). Akademisierung der Psychotherapie: Aktuelle Entwicklungen, historische Annäherungen und internationale Perspektiven. Wien: Facultas.

Duncan, B. (2010). On becomig a better therapist. Psychotherapy in Australia, 16, 42-51.

Elliger, T. (Hrsg.). (1986). S. Freud und die akademische Psychologie. Ein Beitrag zur Rezeptionsgeschichte der Psychoanalyse in der deutschen Psychologie (1895-1945). Weinheim: Deutscher Studienverlag/Beltz.

European Association of Psychotherapy (2013). The final set of core competencies of the EAP's project to develop the professional competencies of a European psychotherapist. www.psychotherapy-competency.eu.Zugegriffen: 11. Februar 2021.

Felt, U., Nowotny, H., \& Taschwer, K. (1995). Wissenschaftsforschung. Eine Einführung(S. 59). Frankfurt:Campus.

Firbas, W. (2001). Psychotherapie - Mangelerscheinung im Medizinstudium? Wien Klin Wochenschr, 113,395-396.

Fischer, G. (2011). Psychotherapiewissenschaft: Einführung in eine neue humanwissenschaftliche Disziplin. Gießen: Psychosozial-Verlag. Unter Mitarbeit von Christiane Eichenberg

Fonagy, P., \& Kächele, H. (2009). Psychoanalysis and otherlongterm dynamic psychotherapies. In M.-G. Gelder, J. J. Lopez-Ibor \& N. Andreasen (Hrsg.), New Oxford textbook of psychiatry(Bd.2, S. 1337-1349). Oxford: Oxford University Press. 
Fonagy, P., \& Target, M. (1997). Attachment and reflective function: their role in self-organization. Development and psychopathology, 9(4), 679-700.

Fonagy, P., Rost, F., Carlyle, J., McPherson, S., Thomas, R., Pasco Fearon, R. M., Goldberg, D., \& Taylor, D. (2015). Pragmatic randomized controlled trial of long-term psychoanalytic psychotherapy for treatment-resistant depression: the Tavistock Adult Depression Study (TADS). World Psychiatry, 14(3), 312-321.

Gesundheit Österreich GmbH (GÖG) (2020). Analyse der Versorgungswirksamkeit von PsychotherapeutInnen in freier Praxis. Arbeitsfassung, im Druck

Grabenhofer-Eggerth, A., \& Sator, M. (2019). Psychotherapie als Sozialversicherungsleistung - Inanspruchnahme und Finanzierung. Wien: Gesundheit Österreich GmbH.

Hierdeis, H., \& Scherer, M. (2018). Psychoanalyse und Medizin: Perspektiven, Differenzen, Kooperationen.Göttingen: Vandenhoeck\&Ruprecht.

Hochgerner, M. (Hrsg.). (2020a). Grundlagen der Psychotherapie(2.Aufl.).Wien: Facultas.

Hochgerner, M. (2020b). Psychotherapeutische Diagnostik. In M. Hochgerner (Hrsg.), Grundlagen der Psychotherapie 2. Aufl. Wien: Facultas.

Hochgerner, M. (2020c). Eine Übersicht über den Mindestumfang der Curricula zur psychotherapeutischen Ausbildung nach dem österreichischen Psychotherapiegesetz. Unpubliziertes Manuskript.

Höfner, C., \& Hochgerner, M. (Hrsg.). (2020). Psychotherapeutische Diagnostik. Wien, New York: Springer.

Jones, E. E. (2000). Therapeutic action: a guide to psychoanalytic therapy. Northvale: Jason Aronson.

Kadi, B. U., Ruhs, A., \& Löffler-Stastka, H. (2012). An den Grenzen der Disziplin [At the limits of discipline]. Neuropsychiatrie, 26(4), 179-186. https://doi.org/10.1007/s40211012-0029-3.

Kierein, M., Pritz, A., \& Sonneck, G. (1991). Psychologengesetz/ Psychotherapiegesetz. Kurzkommentar. Wien: Orac.

Kohl, S., Barnow, S., Brähler, E., Fegert, J. M., Fliegel, S., Freyberger, H. J., \& Strauß, B. (2009). Die Psychotherapieausbildung aus Sicht der Lehrkräfte. Psychotherapeut, 54, $445-456$.

Korunka, Ch , \& Hochgerner, M. (2021). Psychotherapie in Österreich: Der Ausbildungswegund die beruflicheSituation von PsychotherapeutInnen. In W. Datler, A. Drossos, Ch Korunka \& E. Gornik (Hrsg.), Akademisierung der Psychotherapie: Aktuelle Entwicklungen, historische Annäherungen und internationale Perspektiven. Wien: Facultas.

Krause, R. (1997). Grundlagen. Bd. 1. Stuttgart, Berlin, Köln: Kohlhammer.

Krause, K., Midgley, N., Edbrooke-Childs, J., et al. (2020). A comprehensive mapping of outcomes following psychotherapy for adolescent depression: the perspectives of young people, their parents and therapists. Eur Child Adolesc Psychiatry. https://doi.org/10.1007/s00787-02001648-8.

Lambert, M. (Hrsg.). (2013). Bergin and Garfield's handbook of psychotherapy and behavior change (5. Aufl.). New Jersey: John Wiley \& Sons.

Lambert, M.J. (Hrsg.). (2004). Bergin and garfield's handbook of psychotherapy and behavior change(4.Aufl.). NewYork: Wiley.

Leichsenring, F. (2004). Randomized controlled vs. naturalistic studies. Anew research agenda. Bulletin of the Menninger Clinic, 68, 137-151.

Leichsenring, F., \& Rabung, S. (2011). Long-term psychodynamic psychotherapy in complex mental disorders: up- date of a meta-analysis. The British Journal of Psychiatry, 199(1), 15-22.

Leichsenring, F., Luyten, P., Hilsenroth, M. J., Abbass, A., Barber J. P., Keefe, J. R., Leweke, F., Rabung, S., \&Steinert, C. (2015). Psychodynamic therapymeets evidence-based medicine: a systematic review using updated criteria. The Lancet Psychiatry, 2(7), 648-660.

Leitner, A., Schigl, B., \& Märtens, M. (2014). Wirkung, Risiken und Nebenwirkungen von Psychotherapie. Ein Beipackzettel für TherapeutInnen und PatientInnen. Wien: Facultas.

Leuzinger-Bohleber, M., Kaufhold, J., Kallenbach, L., Negele, A., Ernst, M., Keller, W., Fiedler, G., Hautzinger, M., Bahrke, U., \& Beutel, M. (2019). How to measure sustained psychic transformations in long-term treatments of chronically depressed patients: symptomatic and structural changes in the LAC depression study of the outcome of cognitive-behavioural and psychoanalytic long-term treatments. The International Journal of Psychoanalysis, 100(1), 99-127.

Leuzinger-Bohleber, M., Stuhr, U., Rüger, B., \& Beutel, M. (2003). How to study the quality of psychoanalytic treatments' and their long-term effects on patients' well-being. International Journal of Psychoanalysis, 84, 263-290.

Löffler-Stastka, H., Blüml, V., \& Bös, C. (2010). Exploration of personalityfactorsand theirimpact on therapyutilization: the externalizing mode of functioning. Psychotherapy Research, 20, 295-308.

Löffler-Stastka, H., Gelo, O., Pleschberger, I., Schröder, T., Orlinsky, D.E., Rønnestad, M.H., \& Willutzki, U. (2018). Psychotherapieausbildung in Österreich: Basisdaten und soziodemographische Hintergrunddaten aus der SPRISTAD-Studie. Psychopraxis. Neuropraxis, 21(5), 227-231.https://doi.org/10.1007/s00739-018-0508-9.

Löffler-Stastka, H., Gelo, O., Pleschberger, I., Hartmann, A., Orlinsky, D.E., Rønnestad, M.H., \& Willutzki, U. (2019). Psychotherapie-Berufsausbildung in Österreich: Basis- und soziodemografische Hintergrunddaten aus einer SPRISTAD-Pilotstudie (Society of Psychotherapy Research Interest Section on Therapist Training and Development) [Psychotherapy training in Austria. Baseline and socio-demographic background data from a SPRISTAD (Society of Psychotherapy Research Interest Section on Therapist Training and Development) - Pilotstudy]. Zeitschrift für Psychosomatische Medizin und Psychotherapie, 65, 341-352. https://doi.org/10.13109/zptm.2019.65. 4.341 .

Löffler-Stastka, H., Rössler-Schülein, H., \& Skale, E. (2008). Therapie-Abbruch-Prädiktoren in psychoanalytischen Behandlungen von Persönlichkeitsstörungen. Zeitschrift für Psychosomatische Medizin und Psychotherapie, 54, 63-76.

Lorentzen, S., Rønnestad, M. H., \& Orlinsky, D. (2011). Sources ofinfluence on the professional development of psychologists and psychiatrists in Norway and Germany. European Journal of Psychotherapy \& Counselling, 13(2), 141-152.

Luborsky, L. (1995). Are common factors across different psychotherapies the main explanation for the Dodo bird verdict that "everybody has won so all shall have prizes"? Clinical Psychology: Science and Practice, 2, 106-109.

Luborsky, L., Singer, B., \& Luborsky, L. (1975). Comparative studies of psychotherapies: is it true that "everybody has won and all must have prizes"? Archives of General Psychiatry, 32, 995-1008.

Luyten, P., \& Blatt, S. J. (2013). Interpersonal relatedness and self-definition in normal and disrupted personality devel- 
opment: Retrospect and prospect. American Psychologist, 68(3), 172-183. https:// doi.org/10.1037/a0032243.

DeMaat, S., deJonghe, F., deKraker, R.,Leichsenring, F., Abbass, A., Luyten, P., Barber, J., Rien, V., \& Dekker,J. (2013). Current state of the empirical evidencefor psychoanalysis: a metaanalytic approach. Harvard Rev Psychiat, 21(3), 107-137.

De Maat, S., de Jonghe, F., Schoevers, R., \& Dekker, J. (2009). The effectiveness of long-term psychoanalytic therapy: a systematic review of empirical studies. Harvard Rev Psychiat, 17(1), 1-23.

De Maat, S., Philipszoon, F., Schoevers, R., Dekker, J., \& De Jonghe, F. (2007). Costs and benefits of long-term psychoanalytic therapy: changes in health care use and work impairment. Harvard Rev Psychiat, 15(6), 289-300.

Margraf, J., \& Brengelmann, J.C. (1992). Die TherapeutPatient-Beziehung in der Verhaltenstherapie. München: Röttger.

Nissen-Lie, H.A., Havik, O. E., Høglend, P.A., Monsen, J.T., \& Rønnestad, M.H. (2013). A contribution of the quality of therapists' personal lives to the development of the working alliance. J Couns Psychol, 60, 483-495.

Nitzschke, B. (Hrsg.). (1989). Freud und die akademische Psychologie. Kritische Beiträge zu einer historischen Kontroverse. München: Psychologie Verlags Union.

Norcross, J.C. (2002). Psychotherapy relationships that work: Therapist contributions and responsiveness to patients. New York: Oxford University Press.

Norcross, J. C., \& Lambert, M. J. (2019). Evidence-based therapist contributions. Psychotherapyrelationships that work, Bd. 1. Oxford: Oxford University Press.

Norcross, J.C., \& Wampold, B.E. (2019). Evidence-based therapist responsiveness. Psychotherapy relationships that work, Bd. 2. Oxford: Oxford University Press.

Norcross, J.C., VandenBos, G. R., \& Freedheim, D. K. (Hrsg.). (2011). History of psychotherapy: continuity and change (2. Aufl.). Washington: American Psychological Association. https://doi.org/10.1037/12353-000.

ÖGPPM Psy-Diplome. Elektronisch abrufbar auf der Homepage der Österreichischen Gesellschaft für Psychosomatik und Psychotherapeutische Medizin (ÖGPPM). http:// www.oegppm.at/default3.asp?active_page_id=361\&par ent_page_id=322\&pparent_page_id=1. Zugegriffen: 17 . Juni2020.

Orlinsky, D., \& Howard, K. (1986). Process and outcome in psychotherapy. In S.L. Garfield \& A.E. Bergin (Hrsg.), Handbook of psychotherapy and behavior change (3. Aufl. S.311-384). New York: Wiley.

Orlinsky, D. E., \& Rønnestad, M.H. (2005). How psychotherapists develop: A study of therapeutic work and professional development. Washington DC: American PsychologicalAssociation.

Orlinsky, D. E., Grawe, K., \& Parks, B. K. (1994). Process and outcome in psychotherapy: Noch einmal. In A.E. Bergin \& S. L. Garfield (Hrsg.), Handbook of psychotherapy and behavior change (4. Aufl. S. 270-376). New York:Wiley.

Orlinsky, D. E., Strauss, B., Rønnestad, M.H., Hill, C., Castonguay, L., Willutzki, U., \& Carlsson, J. (2015). Education and training. A collaborative study of development in psychotherapy trainees. Psychotherapy Bulletin, 50,21-25.

Österreichische Ärztekammer (ÖÄK) Diplomrichtlinie Psychotherapeutische Medizin (Verordnung 2018). https:// www.arztakademie.at/diplome-zertifikate-cpds/oeaekdiplome/psychoth-medizin/.Zugegriffen: 16. Juni 2020.

Pawlowsky, G. (2018). Zur Entwicklung der Psychotherapie in Österreich. In M. Hochgerner (Hrsg.), Grundlagen der Psychotherapie(S.635-648). Wien: Facultas.
Pawlowsky, G. (2020). Ergänzung zur 2. Auflage. In M. Hochgerner (Hrsg.), Grundlagen der Psychotherapie Bd. 2. Wien: Facultas.

Pawlowsky, G. (2021). Eine Analyse der Quellenberufe der eingetragenen Psychotherapeutinnen und Psychotherapeutenin Österreich. Unveröffentlichtes Manuskript.

Riess, G. (2018). Praxisorientierte Psychotherapieforschung. Leitfaden zur Förderung von Wissenschaft und Forschung in der psychotherapeutischen Ausbildung. Wien: Gesundheit Österreich GmbH.

Rudolf, G. (1991). Die therapeutische Arbeitsbeziehung. Heidelberg: Springer.

Scheidt, C. (Hrsg.). (1986). Die Rezeption der Psychoanalyse in der deutschsprachigen Philosophie vor 1940. Frankfurt: Suhrkamp.

Schmidbauer, W. (2012). Die Geschichte der Psychotherapie. Von der Magie zur Wissenschaft. München:Herbig.

Seitz, T., Stastka, K., Schiffinger, M., Turk, B.R., \& LöfflerStastka, H. (2019). Interprofessional care improves healthrelated well-being and reduces medical costs for chronic pain patients. Bulletin of the Menninger Clinic, 83(2), 105-127.https://doi.org/10.1521/bumc_2019_83_01.

Shedler, S. (2010). The efficacy of psychodynamic psychotherapy. American Psychologist, 65, 98-109.

Slunetzko, Th (2009). Psychotherapie. Eine Einführung. Wien: Facultas.

Springer-Kremser, M., Eder, A., Jandl-Jager, E., \& Hager, I. (2002). Can legislation provide a better match between demand and supply in psychotherapy? Soc Psych Psych Epid, 37, 492-500.

Steinert, C., Munder, T., Rabung, S., Hoyer, J., \& Leichsenring, F. (2017). Psychodynamic therapy: as efficacious as other empirically supported treatments? A meta-analysis testing equivalence of outcomes. American Journal of Psychiatry, 174(10), 943-953.

Steinmair, D., Richter, F., \& Löffler-Stastka, H. (2020). Relationship between mentalizing and working conditions in health care. Int. J. Environ. Res. Public Health, 17(7), 2420. https://doi.org/10.3390/ijerph17072420.

Stiles, W. B., Barkham, M., Mellor-Clark, J., \& Connell, J. (2008). Effectiveness of cognitive-behavioural, person-centred, and psychodynamic therapiesinUKprimary-careroutine practice: Replication in a larger sample. Psychological Medicine, 38, 677-688.

Strauß, B., \& Kohl, S. (2009). Themen der Ausbildungsforschung in der Psychotherapie. Psychotherapeut, 54, 411-426.

Stumm, G., \& Pritz, A. (Hrsg.). (2007). Wörterbuch der Psychotherapie. Wien, New York: Springer.

Sudak, D. M., \& Goldberg, D.A. (2012). Trends in psychotherapy training: a national survey of psychiatry residency training. Acad Psychiatr, 36, 369-373.

Taubner, S., Klasen, J., Hanke, W., \& Möller, H. (2015). Ein empirischer Zugang zur Erfassung der Kompetenzentwicklung von PsychotherapeutInnen in Ausbildung. Psychother Forum, 20, 47-53.

Union Europeenne des Medecins Specialistes (UEMS) Charter on training of medical specialists in the EU. Training requirementsforthespeialtyofpsychiatry-UEMS2017/18. www.uems.eu..Zugegriffen: 16. Juni 2020.

Wampold, B.E., \& Imel, Z.E. (2015). The great psychotherapy debate: the evidence for what makes psychotherapy work. NewYork: Routledge.

Westen, D. (1998). The scientific legacy of Sigmund Freud: toward a psychodynamically-informed psychological science. Psychological Bulletin, 124,333-371. 
Westen, D., Novotny, C. M., \& Thompson-Brenner, H. (2004). The empirical status of empirically supported psychotherapies: assumptions, findings, and reporting in controlled clinical trials. Psychological Bulletin, 130(4), 631.

Wininger, M. (2011). Steinbruch Psychoanalyse? Zur Rezeption der Psychoanalyse in der akademischen Pädagogik des deutschen Sprachraums zwischen 1900-1945. Schriftenreihe der DGfE-Kommission Psychoanalytische Pädagogik, Bd. 3. Opladen: Budrich.

Wißgott, N. (2009). Die Interessenskonflikte bei der Entstehung des österreichischen Psychotherapiegesetzes. http://www.dr.wissgott.at/MEDIA/MastertheseDr. NorbertWissgott.pdf Master Thesis zur Erlangung des akademischen Grades Master of Science im Universitätslehrgang Psychotherapeutische Medizin an der DonauUniversität Krems. Zugegriffen: 11. Februar 2021.

Zimmermann, J., Löffler-Stastka, H., Huber, D., Klug, G., Alhabbo, S., Bock, A., \& Benecke, C. (2015). Is it all about the higher dose? Why psychoanalytic psychotherapy is an effective treatment for major depression. Clinical Psychology \& Psychotherapy, 22, 469-487.https:// doi.org/10. 1002/cpp.1917.

Hinweis des Verlags Der Verlag bleibt in Hinblick auf geografische Zuordnungen und Gebietsbezeichnungen in veröffentlichten Karten und Institutsadressen neutral. 